\title{
Surface plasmon resonance in nanostructured metal films under the Kretschmann configuration
}

\author{
Hai-Sheng Leong, ${ }^{1}$ Junpeng Guo, ${ }^{1, a)}$ Robert G. Lindquist, ${ }^{1}$ and Qing H. Liu ${ }^{2}$ \\ ${ }_{1}^{1}$ Department of Electrical and Computer Engineering, University of Alabama in Huntsville, Huntsville, \\ Alabama 35899, USA \\ ${ }^{2}$ Department of Electrical and Computer Engineering, Duke University, Durham, North Carolina 27708, \\ $U S A$
}

(Received 16 June 2009; accepted 15 November 2009; published online 28 December 2009)

\begin{abstract}
We systematically investigated the surface plasmon resonance in one-dimensional (1D) subwavelength nanostructured metal films under the Kretschmann configuration. We calculated the reflectance, transmittance, and absorption for varying the dielectric fill factor, the period of the 1D nanostructure, and the metal film thickness. We have found that the small dielectric slits in the metal films reduce the surface plasmon resonance angle and move it toward the critical angle for total internal reflection. The reduction in surface plasmon resonance angle in nanostructured metal films is due to the increased intrinsic free electron oscillation frequency in metal nanostructures. Also we have found that the increasing the spatial frequency of the 1D nanograting reduces the surface plasmon resonance angle, which indicates that less momentum is needed to match the momentum of the surface plasmon-polariton. The variation in the nanostructured metal film thickness changes the resonance angle slightly, but mainly remains as a mean to adjust the coupling between the incident optical wave and the surface plasmon-polariton wave. (C) 2009 American Institute of Physics. [doi:10.1063/1.3273359]
\end{abstract}

\section{INTRODUCTION}

Surface plasmons (SPs) are the collective free electron density oscillations on surfaces of metals in contact with dielectric materials. ${ }^{1}$ The coupling of SPs with the electromagnetic field near the metal-dielectric interface gives rise to the surface plasmon polariton (SPP). SPPs can propagate along the metal-dielectric interface as guided electromagnetic $\operatorname{modes}^{1}$ and can also be localized near metallic nanostructures such as nanoparticles and nanorods due to the confinement of free electrons in these nanostructures. Free electron density oscillations in metallic nanostructures coupled with confined electromagnetic fields are called localized SPPs (LSPP). ${ }^{1}$ LSPP can be excited by tuning the excitation optical frequency to the LSPP resonance frequency where energy transfers happen between impinging photons and localized plasmon polaritons. To excite propagating SPPs, the additional momentum is required to overcome the momentum mismatch between the impinging photons and the SPPs. The most common configurations used to achieve the momentum match are the Kretschmann and Raether, ${ }^{2}$ Otto, ${ }^{3}$ and grating ${ }^{4,5}$ configurations. In the Kretschmann and Otto configurations, high index prisms are used to increase the momentum of the incident photons. In the grating configuration, the required momentum is provided by the $\mathrm{k}$-vector of the grating.

It has been known that periodic gratings can provide the required momentum to excite the propagating SPPs and also perturb the SPPs because the periodicity of gratings splits dispersion curves into multiple bands. ${ }^{1}$ The dispersion rela-

\footnotetext{
a) Author to whom correspondence should be addressed. Electronic mail: jguo@eng.uah.edu.
}

tion of the perturbed SPPs in sinusoidal metallic gratings ${ }^{6}$ and subwavelength Gaussian grooves ${ }^{7,8}$ have been investigated with an analytical model based upon the method of Chandezon et al. ${ }^{9}$ The study of the dispersion relation for SPPs in optically thick lamellar transmission gratings have shown that localized SPP modes can be excited within the nanoslits. ${ }^{10}$ The dispersion relation of a semi-infinite thick sinusoidal metallic grating in the Otto configuration was studied by Masale ${ }^{11}$ and the dispersion relation of the SPP in thin metal films which are corrugated on both sides, has been investigated by Schröter and Heitmann. ${ }^{12}$ The study of SPPs in periodic nanograting structures serves many purposes. One of them is for enhancing the sensitivity of biochemical sensors. It has been reported that sixfold sensitivity enhancement can be achieved by using partially etched periodic nanostructures on metal films while it was operated near the edge of SP band gap. ${ }^{13}$ The enhancement of sensitivity in partially etched subwavelength rectangular grating on metal film surfaces also has been investigated and reported. ${ }^{14,15}$

In this paper, we investigated the SP resonance in onedimensional (1D) nanostructured gold films under the Kretschmann configuration. The 1D nanostructured metal films are rectangular subwavelength gratings etched through gold metal films with pitch and thickness smaller than the wavelength. To our knowledge, this is the first systematic investigation of SP resonance in completely etched 1D grating nanostructure gold metal films. Because the period of the 1D nanostructures is smaller than the wavelength, the nanostructured gold films can be regarded as effective materials. ${ }^{16-18}$ The findings in this work provide physical insight how nanostructured metal films affect SP resonance, and can be potentially useful for designing better nanostructured metal film SP resonance biochemical sensors. 


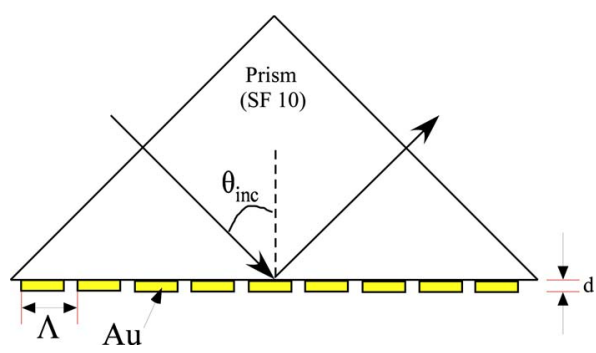

FIG. 1. (Color online) In the Kretschmann configuration, nanostructured gold film is placed on the base of a high index prism. The nanoslits between the metal nanogrids and below are filled with same dielectric medium with the index of refraction of 1.333 .

\section{THE DEVICE STRUCTURE}

Our device structure is a rectangular metal nanograting that is placed directly on top of a prism with no intermediate metal layer in between nanostructured metal film and the prism. Light is incident upon the nanostructured metal film through a high refractive index prism, as shown in Fig. 1. The nanostructured metal film has the following parameters: the thickness of the nanostructured film $(d)$ and the period of the grating $(\Lambda)$. The fill factor, $f$, is defined as the ratio of the slit width to the period of the grating. The slits are filled with a dielectric material. In this paper, we assume that the nanostructured gold films are illuminated with a transverse magnetic (TM) polarized $850 \mathrm{~nm}$ wavelength laser at the incidence angle, $\theta_{\text {inc }}$. The refractive index of the prism is 1.723 , the index of refraction of SF10 glass. The refractive index for the dielectric material in the nanoslits and below is 1.333 . The electric permittivity of gold at the $850 \mathrm{~nm}$ wavelength is $-24.830-2.239 i$ from Ref. 19.

\section{CALCULATION RESULTS}

In this work, we calculate the reflection, transmission, and absorption of the nanostructured gold metal films versus the angle of incidence. The numerical technique used for calculating the reflectance, transmittance, and absorption from the nanostructured film is the well-known Fourier modal method. ${ }^{20-26}$ The technique is also called rigorous coupled wave approach, which expands the fields and the electrical permittivity into a Fourier series with a large finite number of modes. The coefficients of the fields are obtained by solving the eigenvalue problem and satisfying the boundary conditions. Use of this method to calculate the periodic metallic nanostructures have been successfully confirmed with experiment. ${ }^{27-30}$ The convergence of our numerical results is validated with 121 Fourier modes retained in our calculations.

Figure 2 shows the calculated reflectance, transmittance, and absorption versus the angle of incidence from (a) a uniform gold film and (b) a nanostructured gold film, respectively. Absorption here is defined as the total energy loss of the system, $A=1-R-T$, where $A, T$, and $T$ represents the absorption, reflectance, and transmittance, respectively. Both the uniform and nanostructure gold thin films are $50 \mathrm{~nm}$ thick and they are illuminated with a TM-polarized $850 \mathrm{~nm}$ wavelength laser light. The period of the rectangular metal lines completely etched in gold film is $200 \mathrm{~nm}$, which is
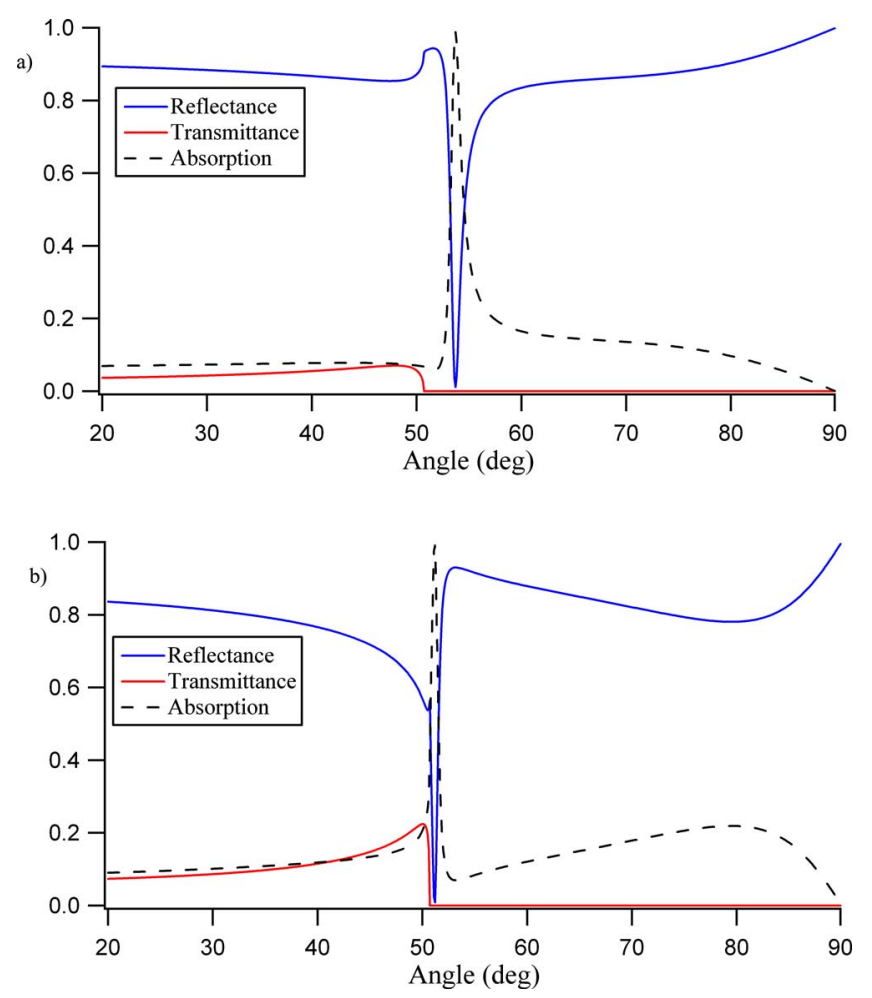

FIG. 2. (Color online) Reflectance, transmittance, and absorption as functions of the incidence angle $\theta_{\text {inc }}$ for (a) uniform metal film and (b) nanostructured metal film with $f=0.2$. The solid blue and red curves represent the reflectance and transmittance respectively, and the dashed curve represents the absorption in the metal films.

much smaller than the wavelength, and the width of the dielectric filled slits is $40 \mathrm{~nm}$. The fill factor, therefore, is 0.2 . For the uniform gold film, the SP resonance happens at the incidence angle of $53.7^{\circ}$. For the nanostructured gold film, the SP resonance angle is $51.2^{\circ}$. The reduction in the SP resonance angle indicates less momentum is required for matching the momentum of the SPP in the nanostructured film. The reduction in the SPP propagation constant can be explained as the localization of free electrons in the metal nanogrids has increased the intrinsic oscillation frequency of these free electrons, which is equivalent to the increase in the bulk plasma frequency in the Drude model. ${ }^{1}$ The increase in the bulk plasma frequency of the effective nanostructured metal results in a more negative real part of the electric permittivity. More negative real part of the electric permittivity gives a smaller SPP propagation constant, which is similar to the phenomenon occurred as shifting the excitation optical frequency to lower frequency. At lower optical frequency, the real part of electric permittivity of the metal has a larger absolute value with the negative sign. Comparing the reflection curve from the nanostructured gold film with that of the uniform film, it can be seen that the reflection curve from the nanostructured film has a sharper resonance than that of the uniform thin film for this small fill factor. This sharper SP resonance is probably due to the smaller SP propagation attenuation along the nanostructured metal film.

\section{A. SP resonance change with the dielectric fill factor}

We fixed the period of the structure at $200 \mathrm{~nm}$ and the metal film thickness at $50 \mathrm{~nm}$ and varied the fill factor of the 

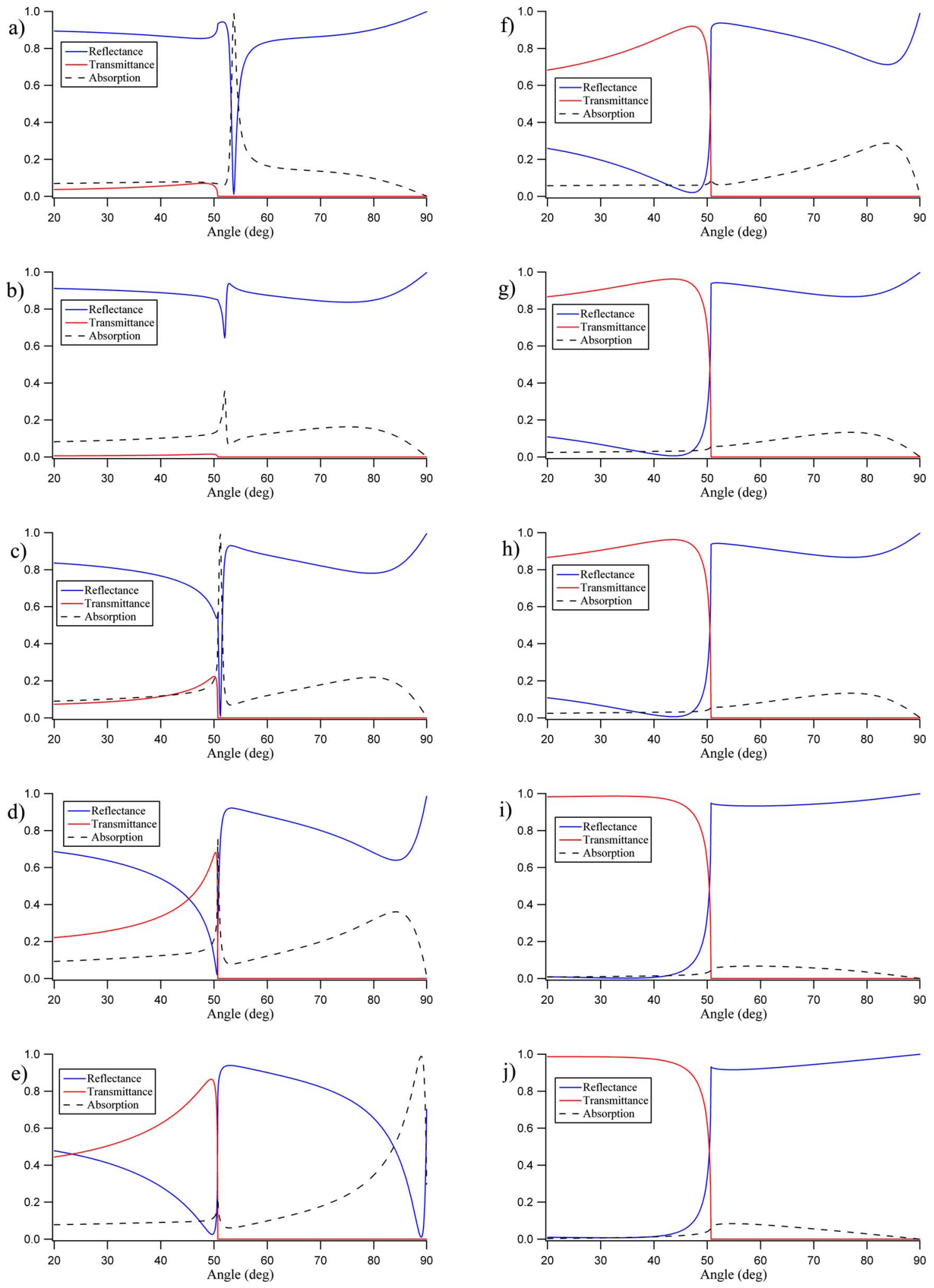

FIG. 3. (Color online) Evolution of the SP resonance in nanostructured gold metal films with the fill factor (a) $f=0$, (b) $f=0.1$, (c) $f=0.2$, (d) $f=0.3$, (e) $f$ $=0.4$, (f) $f=0.5$, (g) $f=0.6$, (h) $f=0.7$, (i) $f=0.8$, and (j) $f=0.9$. The period of the $1 \mathrm{D}$ nanostructure is $200 \mathrm{~nm}$ and the thickness of the metal film is $50 \mathrm{~nm}$.

nanostructure from 0 to 0.9 . The fill factor $(f)$ is the ratio of the slit width over the period $(\Lambda)$. Figures $3(\mathrm{a})-3(\mathrm{j})$ show the reflectance, transmittance, and absorption of the nanostructure thin film versus the angle of incidence for different fill factors varying from 0 to 0.9 . In Fig. 3(a), where $f=0$, the metal film is uniform film. The SP resonance angle is $53.7^{\circ}$. With Fig. 3(a) as the base for comparison, we see how the SP resonance angle changes with the fill factor. It can be seen that the SP resonance shifts to the incidence angles of $52.1^{\circ}$, $51.2^{\circ}$, and $50.7^{\circ}$ for the fill factor $f=0.1,0.2$, and 0.3 , respectively. As the fill factor is further increased, the SP resonance angle approaches to $50.68^{\circ}$, which is the critical angle for total internal reflection between the high index prism and the low index dielectric material on the other side of the 
metal grating.

The shift of SP resonance angle to the smaller angle toward the critical angle of total internal reflection indicates less momentum required to excite SPPs in the nanostructured film. This can be explained as follows: Because the electric field vector is perpendicular to the metal nanogrids, the localization of free electrons in the metal nanogrids increases the intrinsic oscillation frequency of free electrons, which is equivalent to the increase in the bulk plasma frequency in the effective medium in the Drude model. ${ }^{1}$ The increase in the bulk plasma frequency results in a more negative real part of the electric permittivity. More negative real part of the electric permittivity gives a smaller SPP propagation constant, which is similar to reducing the excitation optical frequency to lower frequency. As moving to lower frequency, the real part of electric permittivity of the metal becomes more negative, ${ }^{31}$ and the propagation constant of SPP becomes smaller.

As the fill factor increases further, the total internal reflection dominates and the SP resonance disappears near the critical angle. However, another SP resonance occurs at the large angle of incidence (see $f=0.3,0.4$, and 0.5 ). This SP resonance is probably due to the coupling between the localized SPP and propagating SPP. For the fill factor from 0.6 to 0.9 , the SP resonance is negligible because there are very small amount of metal in the nanostructured films.

\section{B. SP resonance change with the period of the subwavelength nanograting}

We fixed the fill factor of the nanostructured gold metal film at $f=0.2$ and the thickness of the film at $d=50 \mathrm{~nm}$, and calculated the reflectance and absorption versus the angle of incidence for subwavelength nanogratings of period $(\Lambda)$ varying from 100, 150, 200, to $250 \mathrm{~nm}$. Figure 4(a)) shows the calculated reflectance versus the angle of incidence. Figure 4(b)) shows the absorption versus the angle of incidence. It is clearly seen that as the period of the subwavelength grating decreases, the SP resonance occurs at smaller incidence angles. This indicates less momentum is needed to match to the momentum of the SPPs. This can be explained as: when the line-width of nanometal grids is reduced, the intrinsic free electron oscillation frequency in the nanostructures increases. This is equivalent to the increase in bulk plasma frequency in the Drude model. The increase in bulk plasma frequency changes the effective material property, which reduces the SP propagation constant. The strongest SP resonance occurs when the period of the nanograting is 200 $\mathrm{nm}$. This indicates the maximal coupling between the incident photons and SPPs in the nanostructured metal film. As the period increases toward the half of the wavelength, the SP resonance gradually diminishes because of the diminishing coupling between the incident optical wave and SPP wave.

\section{SP resonance change with the thickness of nanostructured metal film}

We fixed the fill factor of the nanostructured metal film at $f=0.2$, and the period of the nanostructure grating $(\Lambda)$ at
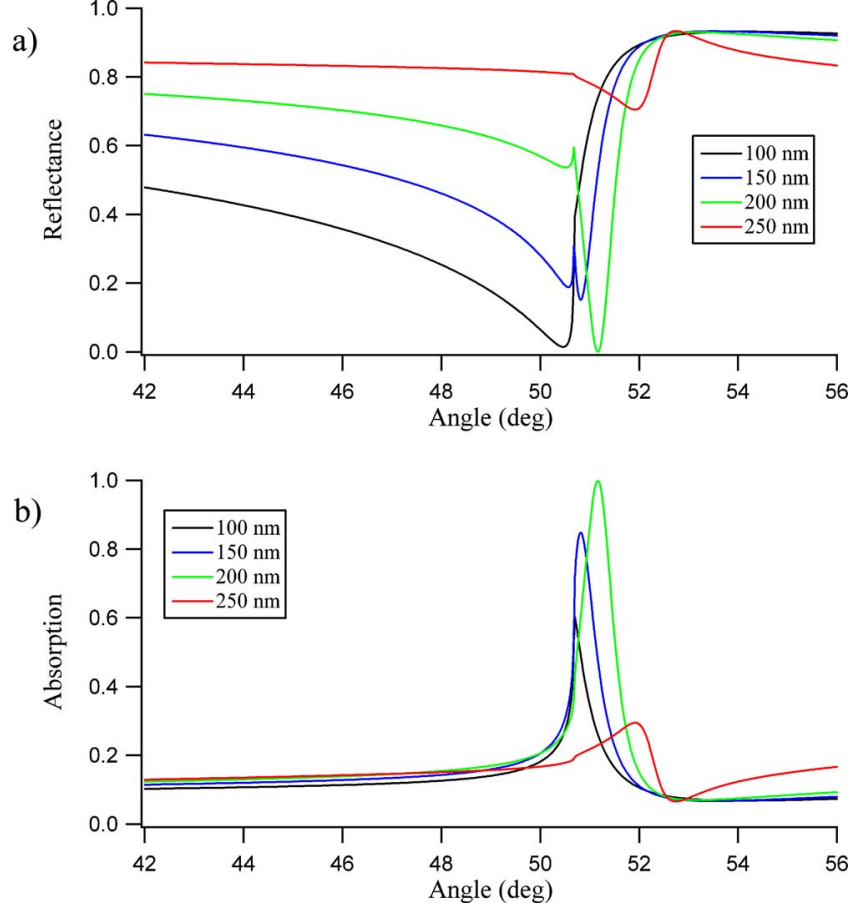

FIG. 4. (Color online) (a) Reflectance and (b) absorption vs the angle of incidence for different periods of 1D nanostructured films. The thickness (d) is $50 \mathrm{~nm}$. The fill factor is 0.2 .

$200 \mathrm{~nm}$, and calculated the reflectance and absorption versus the angle of incidence for the nanostructured film varying the thickness $(d)$ from $d=30,35,40,50$, to $60 \mathrm{~nm}$, Fig. 5(a) shows the calculated reflectance versus the angle of incidence angle. Figure 5(b) shows the absorption versus the incidence angle. The absorption at the incidence angle of $51.2^{\circ}$ appears to be the strongest when the nanostructure

a)

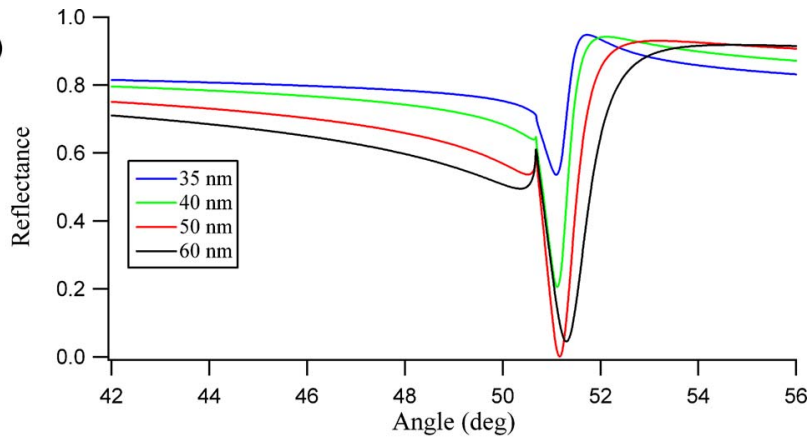

b)

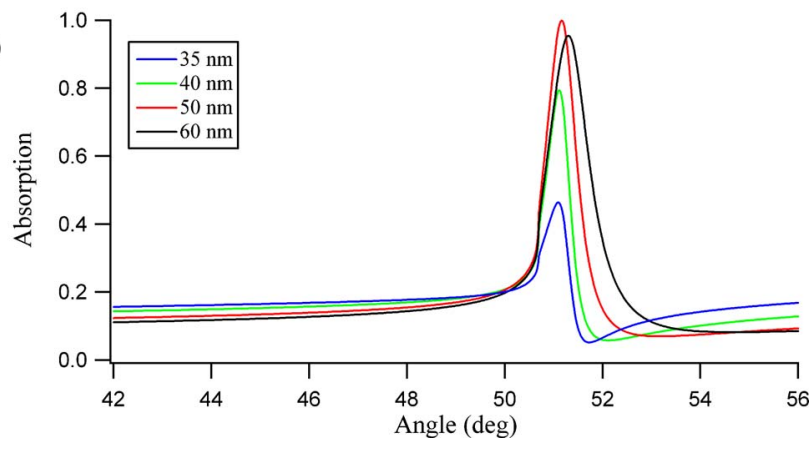

FIG. 5. (Color online) (a) Reflectance and (b) absorption for different thicknesses of nanostructured gold film. The period is $200 \mathrm{~nm}$ and the fill factor is 0.2 . 

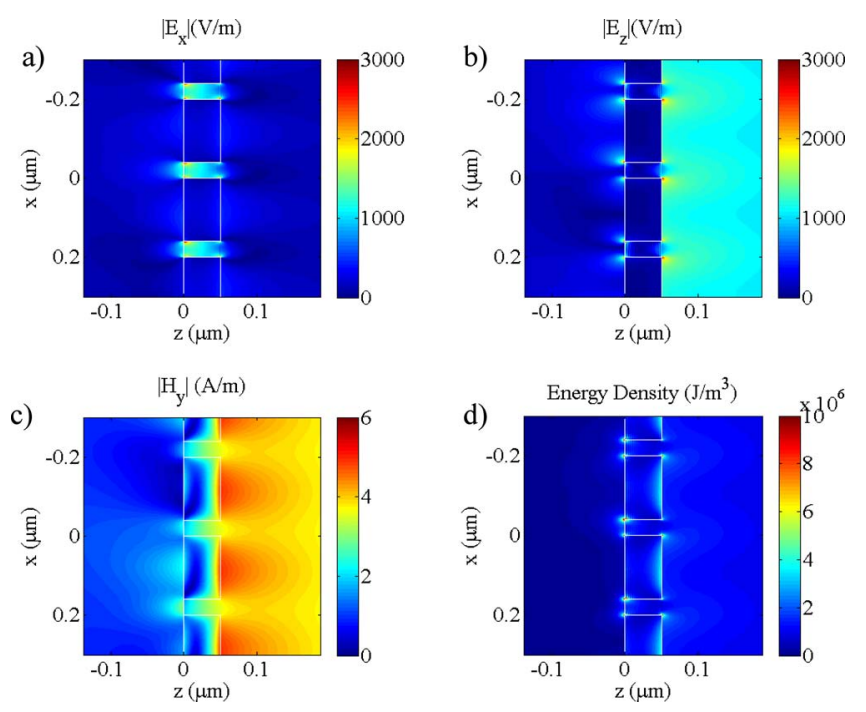

FIG. 6. (Color online) (a)The amplitude of the tangential component ( $x$ axis) of the electric field, (b) the amplitude of the normal component ( $\mathrm{z}$-axis) of the electric field, (c) the amplitude of the magnetic field, and (d) the energy density distribution. Light is incident from the left. The angle of incidence is at the SP resonance angle of $51.2^{\circ}$.

thickness is at $50 \mathrm{~nm}$. This indicates that the maximum energy transfer from the incident photons to SPP occurs at this thickness. Also, it is seen that the SP resonance angle increases slightly as the metal film thickness increases. This is because thicker metal films support more tightly confined propagating SPPs. This trend is same as the situation where the uniform metal film is used for SP resonance. The change in SP resonance angle with metal film thickness is not as dramatic as the change with the period of the nanograting. Therefore, variation in thickness can mainly remain as a mean to adjust the coupling between the incident optical wave and SPP wave.

Figure 6 shows the amplitudes of the electric fields (Ex and $\mathrm{Ez}$ ), the magnetic field (Hy), as well as the electromagnetic energy density $\left(w=(1 / 2) \operatorname{Re}\{d[\omega \varepsilon(\omega)] /(d \omega)\}|E|^{2}\right)$. The light is incident upon the nanostructured thin gold film from the high index of refraction medium in the left at the SP resonance angle of $51.2^{\circ}$. The parameters of the nanostructured metal film are $f=0.2, d=50 \mathrm{~nm}$, and $\Lambda=200 \mathrm{~nm}$. It can be seen that the electric field is strong in the slits of the nanostructured film and is strongest near the edges of the slits. This is because SPP energy is highly localized at near the edges of the nanogrids.

\section{CONCLUSION}

In this paper, we systematically investigated SP resonances in nanostructured metal films under the Kretschmann configuration. We calculated the reflectance, transmittance, and absorption for varying fill factor, the period of the 1D nanostructure gold metal film, and the metal film thickness. We found that small dielectric fill factors shift the SP resonance angle toward the critical angle for total internal reflection. This is due to the increased intrinsic free electron oscillation frequency in localized metal nanostructures. As the fill factor increases further, the total internal reflection dominates near the critical angle. As the period of the subwave- length grating decreases, less momentum is required to match to the momentum of the SPP. This is because the localization of free electrons in nanostructures has increased the intrinsic free electron oscillation frequency, the coupling of localized SP with the propagating SP reduce the propagation constant. The variation in the nanostructured metal film thickness slightly changes the SP resonance with the same trend as in the uniform metal film. But the thickness mainly remains as a mean to adjust the coupling between the incident optical wave and the SPP wave. The findings in this work might be useful for designing better nanostructured metal SP resonance biochemical sensors.

\section{ACKNOWLEDGMENTS}

This work was sponsored by the National Science Foundation under Contract Nos. NSF-0814103 and NSF0813870, and the National Aeronautics and Space Administration (NASA) under Contract No. NNX07AL52A.

${ }^{1}$ H. Raether, Surface Plasmons on Smooth and Rough Surfaces and on Gratings (Springer-Verlag, Berlin, 1988).

${ }^{2}$ E. Kretschmann and H. Raether, Z. Naturforsch. A 23, 2135 (1968).

${ }^{3}$ A. Otto, Z. Phys. 216, 398 (1968).

${ }^{4}$ Y. Y. Teng and E. A. Stern, Phys. Rev. Lett. 19, 511 (1967).

${ }^{5}$ R. H. Ritchie, E. T. Arakawa, J. J. Cowan, and R. N. Hamm, Phys. Rev. Lett. 21, 1530 (1968).

${ }^{6}$ W. L. Barnes, T. W. Preist, S. C. Kitson, and J. R. Sambles, Phys. Rev. B 54, 6227 (1996).

${ }^{7}$ W. C. Tan, T. W. Preist, J. R. Sambles, and N. P. Wanstall, Phys. Rev. B 59, 12661 (1999).

${ }^{8}$ I. R. Hooper and J. R. Sambles, Phys. Rev. B 65, 165432 (2002).

${ }^{9}$ J. Chandezon, M. T. Dupuis, G. Cornnet, and D. Mystre, J. Opt. Soc. Am. 72, 839 (1982).

${ }^{10}$ J. A. Porto, F. J. García-Vidal, and J. B. Pendry, Phys. Rev. Lett. 83, 2845 (1999).

${ }^{11}$ M. Masale, Physica B (Amsterdam) 325, 385 (2003).

${ }^{12}$ U. Schröter and D. Heitmann, Phys. Rev. B 60, 4992 (1999).

${ }^{13}$ C. J. Alleyne, A. G. Kirk, R. C. McPhedran, N. P. Nicorovici, and D. Maystre, Opt. Express 15, 8163 (2007).

${ }^{14}$ K. M. Byun and S. J. Kim, Opt. Express 13, 3737 (2005).

${ }^{15}$ K. M. Byun, M. L. Shuler, S. J. Kim, S. J. Yoon, and D. Kim, J. Lightwave Technol. 26, 1472 (2008).

${ }^{16}$ S. M. Rytov, Sov. Phys. JETP 2, 466 (1956).

${ }^{17}$ J. Elser, V. A. Podolskiy, I. Salakhutdinov, and I. Avrutsky, Appl. Phys. Lett. 90, 191109 (2007).

${ }^{18}$ J. Elser, R. Wangberg, V. A. Podolskiy, and E. E. Narimanov, Appl. Phys. Lett. 89, 261102 (2006).

${ }^{19}$ A. D. Rakić, A. B. Djurišić, J. M. Elazar, and M. L. Majewski, Appl. Opt. 37, 5271 (1998).

${ }^{20}$ H. Kogelnik, Bell Syst. Tech. J. 48, 2909 (1969).

${ }^{21}$ M. G. Moharam and T. K. Gaylord, J. Opt. Soc. Am. 71, 811 (1981).

${ }^{22}$ M. G. Moharam, E. B. Grann, D. A. Pommet, and T. K. Gaylord, J. Opt. Soc. Am. A Opt. Image Sci. Vis 12, 1068 (1995).

${ }^{23}$ M. G. Moharam, D. A. Pommet, E. B. Grann, and T. K. Gaylord, J. Opt. Soc. Am. A Opt. Image Sci. Vis 12, 1077 (1995).

${ }^{24}$ L. Li, J. Opt. Soc. Am. A Opt. Image Sci. Vis 13, 1024 (1996).

${ }^{25}$ T. Tamir and H. C. Wang, IEEE Trans. Microwave Theory Tech. 12, 323 (1964).

${ }^{26}$ C. B. Burckhardt, J. Opt. Soc. Am. 56, 1502 (1966).

${ }^{27}$ K. M. Byun, S. J. Yoon, D. Kim, and S. J. Kim, Opt. Lett. 32, 1902 (2007).

${ }^{28}$ J. Cesario, R. Quidant, G. Badenes, and S. Enoch, Opt. Lett. 30, 3404 (2005).

${ }^{29}$ Y. Kanamori, K. Hane, H. Sai, and H. Yugami, Appl. Phys. Lett. 78, 142 (2001).

${ }^{30}$ S. Park, G. Lee, S. H. Song, C. H. Oh, and P. S. Kim, Opt. Lett. 28, 1870 (2003).

${ }^{31}$ B. Johnson and R. W. Christy, Phys. Rev. B 6, 4370 (1972). 